\title{
Electron microscopy explorations of the human brain: using immunofluorescence to address challenges
}

Kristina Micheva ${ }^{1}$, Marc Perez ${ }^{2}$, Anish Simhal ${ }^{3}$, Richard Weinberg ${ }^{4}$ and Daniel Madison ${ }^{2}$

${ }^{1}$ Stanford University School of Medicine, Stanford, California, United States, ${ }^{2}$ Stanford University School of Medicine, United States, ${ }^{3}$ Autism Center, Child Mind Institute, New York, United States, ${ }^{4}$ University of North Carolina - Chapel Hill, United States

Electron microscopy (EM) of biological material presents unique demands for its application in different species and different tissues. Among the most challenging applications of EM is human brain tissue. The near impossibility of standardizing tissue sources and preparation, as well as the difficulties associated with the large size of the human brain and its variability pose many hurdles for ultrastructural studies, and especially for investigations that require larger number of samples and comparisons between different conditions. Freshly fixed brain tissue from biopsies obtained during neurosurgeries presents the best quality, but even in this case there is large variability from patient to patient and even within samples from the same patient. Application of light microscopy methods for tissue quality screening and subsequent analysis can be used to assist, supplement and expand electron microscopy of human tissue.

Postembedding immunofluorescence is an efficient approach to identify well preserved tissue areas for subsequent EM analysis. For example, immunolabeling for the inhibitory neurotransmitter GABA, which is present in about $20 \%$ of neurons in the brain [1], is very sensitive to the fixation conditions [2] and can be used as an indicator for the quality of fixation. Immunofluorescence is also useful for initial assessment of the ultrastructure quality of specific organelles or subcellular structures. Thus, the myelin sheath surrounding many axons can be difficult to preserve [3], and using immunolabeling for abundant proteins in myelin, like myelin basic protein [4], helps identify problematic samples to be avoided for myelin studies.

Correlative immunofluorescence - scanning EM array tomography [5] further extends human tissue studies to include not only the ultrastructure, but also molecular information. This allows the identification and quantification of diverse synapse types, which can then also be examined for the presence for example, of different postsynaptic receptors. Using this approach, we compare synapses in the human and mouse brain. Because much of our current understanding of the molecular basis of brain function, as well as dysfunction in neuropsychiatric diseases, is based on studies in rodents, such a comparison is essential. We find that synapses in human neocortex are larger and sparser compared to mouse neocortex, but they follow many similar rules to the rodent cortex, such as relative proportions of excitatory and inhibitory synapses, correlations between the sizes of pre- and postsynaptic elements, receptor distributions, synapse type-dependence of mitochondria presence. Pinpointing the similarities and differences between human and mouse brain is essential for the successful application of mouse models towards understanding, preventing and treating human disease [6].

\section{References}

[1] J DeFelipe, Prog Brain Res 136 (2002), p.215.

[2] P Somogyi and AJ Hodgson, J Histochem Cytochem 33 (1985), p.249.

[3] W Möbius, K-A Nave, HB Werner, Brain Res 1641 (2016), p.92.

[4] K Micheva, D Wolman, BD Mensh, E Pax, J Buchanan, SJ Smith, Bock DD, eLife 5 (2016) e15784.

[5] F Collman, J Buchanan, KD Phend, KD Micheva, RJ Weinberg, SJ Smith, J Neurosci 35 (2015) p.5792.

[6] This work was supported by NIH R01 MH111768 and R01 NS094499. 